\title{
Integrated spectral properties of star clusters in the Magellanic Clouds
}

\author{
Andrea V. Ahumada ${ }^{1,2,3}$, M. L. Talavera ${ }^{4}$, J. J. Clariá ${ }^{2,3}$, \\ J. F. C. Santos Jr. ${ }^{5}$, E. Bica ${ }^{6}$, M. C. Parisi ${ }^{2,3}$ and M. C. Torres ${ }^{2}$ \\ ${ }^{1}$ European Southern Observatory - ESO, Chile, email: aahumada@eso.org \\ ${ }^{2}$ Observatorio Astronómico, Universidad Nacional de Córdoba, Argentina \\ ${ }^{3}$ Consejo Nacional de Investigaciones Científicas y Técnicas, CONICET, Argentina \\ ${ }^{4}$ Observatorio Astronómico Centroamericano de Suyapa, UNAH, Honduras \\ ${ }^{5}$ Departamento de Física, ICEx, UFMG, Belo Horizonte, Brazil \\ ${ }^{6}$ Departamento de Astronomia, UFRGS, Porto Alegre, Brazil
}

\begin{abstract}
We present flux-calibrated integrated spectra in the optical spectral range of concentrated star clusters in the Large and Small Magellanic Clouds (LMC-SMC), approximately half of which constitute unstudied objects. We have mainly estimated ages and foreground interstellar reddening values from the comparison of the line strengths and continuum distribution of the cluster spectra with those of template spectra with known parameters. Also reddening values were estimated by interpolation between the extinction maps of Burstein \& Heiles (1982) $(\mathrm{BH})$. A good agreement between ages and reddenings derived through the different procedures was found. The ages of the 27 LMC star clusters range from 5 to $125 \mathrm{Myr}$, while those of the 13 SMC vary from 4 to 350 Myr.
\end{abstract}

Keywords. techniques: spectroscopic, Magellanic Clouds, galaxies: star clusters

\section{Introduction}

The study of extragalactic stellar systems provides relevant information on the star formation and chemical histories of the host galaxies. Despite the multiple observational as well as theoretical projects undertaken in the last few years, our currently existing knowledge of both the stellar formation processes and chemical evolution of galaxies is, in general, incomplete. Even for the galaxies in the Local Group, our present understanding is definitely limited. In this state of affairs, the stellar cluster systems of the Magellanic Clouds (MCs), on account of their proximity, richness, and variety, may furnish us with the ideal ground to conduct a detailed examination of the processes mentioned before. Efforts to create reference spectra of star clusters and grids of their properties to be used as templates for different ages and metallicities in the study of composite stellar populations were made by different authors, e.g., Bica \& Alloin (1986a), Bica (1988), Santos Jr. et al. (1995), and Piatti et al. (2002). The goal of the present study is to collect and analyse a large sample of MCs clusters in view of studying the integrated light properties of such metal deficient clusters; and making them available as template spectra for studies of star clusters in more distant dwarf galaxies.

\section{Cluster sample and observations}

The determination of MCs cluster parameters, particularly age, is fundamental to understand the structure and evolution of these galaxies. Concentrated clusters, with small angular diameter are certainly the most suitable to carry out integrated spectroscopy observations. This is because the cluster as well as the surrounding background regions are 
Table 1. The SMC cluster sample. Cluster identifications are from Lindsay (1958) (L), Kron (1956) (K), Lauberts (1982)(ESO), Westerlund \& Glaspey (1971)(WG), Hodge \& Wright (1974) (HW), Bruck (1976), and Pietrzyński et al. (1998)(OGLE).

\begin{tabular}{lcc}
\hline Cluster & $\alpha_{2000}$ & $\delta_{2000}$ \\
\hline NGC 242, K 22, L 29, SMC_OGLE 18 & $00: 43: 38$ & $-73: 26: 38$ \\
NGC 256, K 23, L 30, ESO29-SC 11, SMC_OGLE 32 & $00: 45: 54$ & $-73: 30: 24$ \\
NGC 265, K 24, L 34, ESO29-SC 14, SMC_OGLE 39 & $00: 47: 12$ & $-73: 28: 38$ \\
B 50 & $00: 49: 03$ & $-73: 22: 00$ \\
K 34, L 53, SMC_OGLE 104 & $00: 55: 33$ & $-72: 49: 58$ \\
IC 1611, K 40, SMC_OGLE 118, L 61, ESO29-SC 27 & $00: 59: 48$ & $-72: 20: 02$ \\
IC 1626, K 53, L 77, ESO29-SC 30 & $01: 06: 14$ & $-73: 17: 51$ \\
IC 1641, HW 62, ESO51-SC 21 & $01: 09: 40$ & $-71: 46: 03$ \\
L 95 & $01: 15: 00$ & $-71: 20: 00$ \\
B 164 & $01: 29: 30$ & $-73: 32: 00$ \\
HW 85 & $01: 42: 00$ & $-71: 17: 00$ \\
WG 1 & $01: 42: 53$ & $-73: 20: 00$ \\
NGC 796, L 115, ESO30-SC 6 & $01: 54: 45$ & $-74: 13: 00$ \\
\hline
\end{tabular}

Table 2. The LMC cluster sample. Cluster identifications are from Lauberts (1982) (ESO), Shapley \& Lindsay (1963) (SL), Kontizas et al. (1990) (KMHK), Pietrzyński et al. (1999) (LMC_OGLE), Hodge \& Sexton (1966) (HS), and Lyngå \& Westerlund (1963) (LW).

\begin{tabular}{|c|c|c|c|}
\hline Cluster & $\alpha_{2000}$ & $\delta_{2000}$ & SWB \\
\hline SL 14, KMHK 28 & $04: 40: 28$ & $-69: 39: 00$ & II \\
\hline NGC 1695, KMHK 101, SL 40 & $04: 47: 44$ & $-69: 22: 00$ & III \\
\hline SL 56, KMHK 142 & $04: 50: 32$ & $-70: 04: 00$ & II \\
\hline SL 58, KMHK 153 & $04: 50: 59$ & $-69: 38: 00$ & III \\
\hline SL 79, KMHK 213 & $04: 52: 53$ & $-71: 39: 00$ & III \\
\hline SL 76, KMHK 206 & 04:53:09 & $-68: 12: 00$ & III \\
\hline NGC 1732, KMHK 209, SL 77 & $04: 53: 11$ & $-68: 39: 00$ & II \\
\hline SL 116, KMHK 315 & $04: 56: 24$ & $-68: 48: 00$ & II \\
\hline SL 168, KMHK 418 & 05:00:44 & $-65: 27: 00$ & III \\
\hline NGC 1822, KMHK 513, SL 210 & 05:05:08 & $-66: 12: 00$ & II \\
\hline HS 109, LMC_OGLE 82 & 05:05:37 & $-68: 43: 06$ & II \\
\hline SL 234, LMC_OGLE 113 & $05: 06: 54$ & $-68: 43: 08$ & II \\
\hline SL 255, KMHK 573 & $05: 07: 55$ & $-70: 03: 00$ & II \\
\hline NGC 1887, KMHK 700, SL 343 & 05:16:05 & $-66: 19: 00$ & II \\
\hline SL 364, KMHK 736 & $05: 17: 41$ & $-71: 03: 00$ & II \\
\hline SL 360, LMC_OGLE 328 & $05: 18: 11$ & $-69: 13: 06$ & 0 \\
\hline SL 386, KMHK 770 & 05:19:50 & $-65: 23: 00$ & II \\
\hline NGC 1944, KMHK 836, SL 426, ESO33-SC 7 & $05: 21: 57$ & $-72: 29: 00$ & III \\
\hline SL 463, KMHK 889, LW 213 & $05: 26: 15$ & $-66: 03: 00$ & II \\
\hline SL 477, KMHK 911 & $05: 26: 23$ & $-71: 41: 00$ & II \\
\hline NGC 1972, LMC_OGLE 481, SL 480 & $05: 26: 48$ & $-69: 50: 17$ & II \\
\hline NGC 2000, KMHK 932, SL 493 & $05: 27: 30$ & $-71: 52: 00$ & II \\
\hline NGC 1986, LMC_OGLE 496, SL 489 & $05: 27: 38$ & $-69: 58: 14$ & II \\
\hline SL 566, KMHK 1061 & $05: 32: 50$ & $-70: 47: 00$ & II \\
\hline NGC 2053, KMHK 1154, SL 623 & $05: 37: 40$ & $-67: 24: 00$ & II \\
\hline SL 763 , KMHK 1448 & $05: 52: 53$ & $-69: 47: 00$ & II \\
\hline NGC 2140, KMHK 1511, SL 773 & $05: 54: 17$ & $-68: 36: 00$ & II \\
\hline
\end{tabular}

well sampled along the slit. Besides, the angular diameter requirement results from the fact that the cluster integrated spectrum must reflect the synthesis of its stellar content. In this study we have selected relatively populous and compact MCs clusters to allow good star sampling in the integrated spectra. The observed SMC star cluster sample is given in Table 1, where their designations in different catalogues are provided, while Table 2 shows the LMC cluster sample including also the SWB type (Searle et al. 1980). 


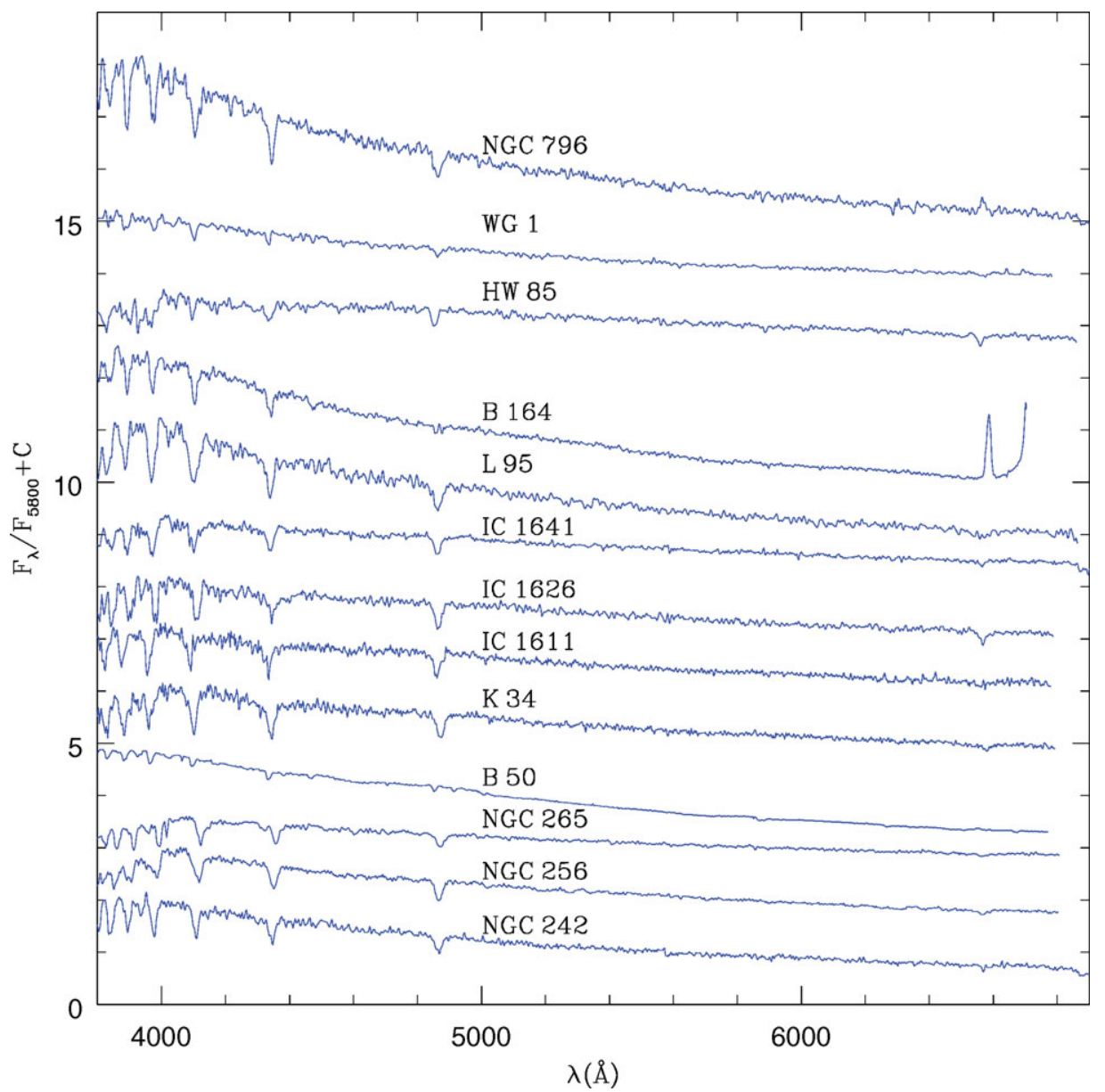

Figure 1. Observed integrated spectra of $13 \mathrm{SMC}$ clusters. Spectra are in relative $F_{\lambda}$ units normalised at $\lambda \sim 5800 \AA$. Constants have been added to the spectra for clarity, except for the bottom one.

All the observations were carried out with the 'Jorge Sahade' $2.15 \mathrm{~m}$ telescope at the Complejo Astronómico El Leoncito (CASLEO, San Juan, Argentina) in several runs. We employed a CCD camera containing a Tektronix chip of $1024 \times 1024$ pixels attached to a REOSC spectrograph (simple mode). The slit was oriented in the east-west direction and the observations were performed scanning the slit across the objects in the north-south direction in order to get a proper sampling of cluster stars. A grating of 300 grooves $/ \mathrm{mm}$ was used. The spectral coverage was the visible range: $\sim(3800-6900) \AA$, with an average dispersion in the observed region of $\sim 140 \AA / \mathrm{mm}(3.46 \AA / \mathrm{pix})$. The slit width was $4.2^{\prime \prime}$, resulting in a resolution of $\sim 14 \AA$, as measured by the mean full width half maximum of the comparison lines. At least two exposures of 20 minutes of each object were taken, depending on the star concentration of the cluster. Standard stars were also observed for flux calibrations, and comparison lamp exposures were taken for wavelength calibration.

In Figure 1 we present the flux-calibrated integrated spectra of the SMC observed clusters, while Figure 2 shows part of the 27 LMC integrated spectra. The whole LMC sample will be presented elsewhere. All the spectra are in relative flux units, normalised to $F_{\lambda}=1$ at $\lambda \sim 5800 \AA$. The spectral lines and different slopes of the continuum energy distributions in both figures are primarily the result of age effects. 
A. V. Ahumada et al.

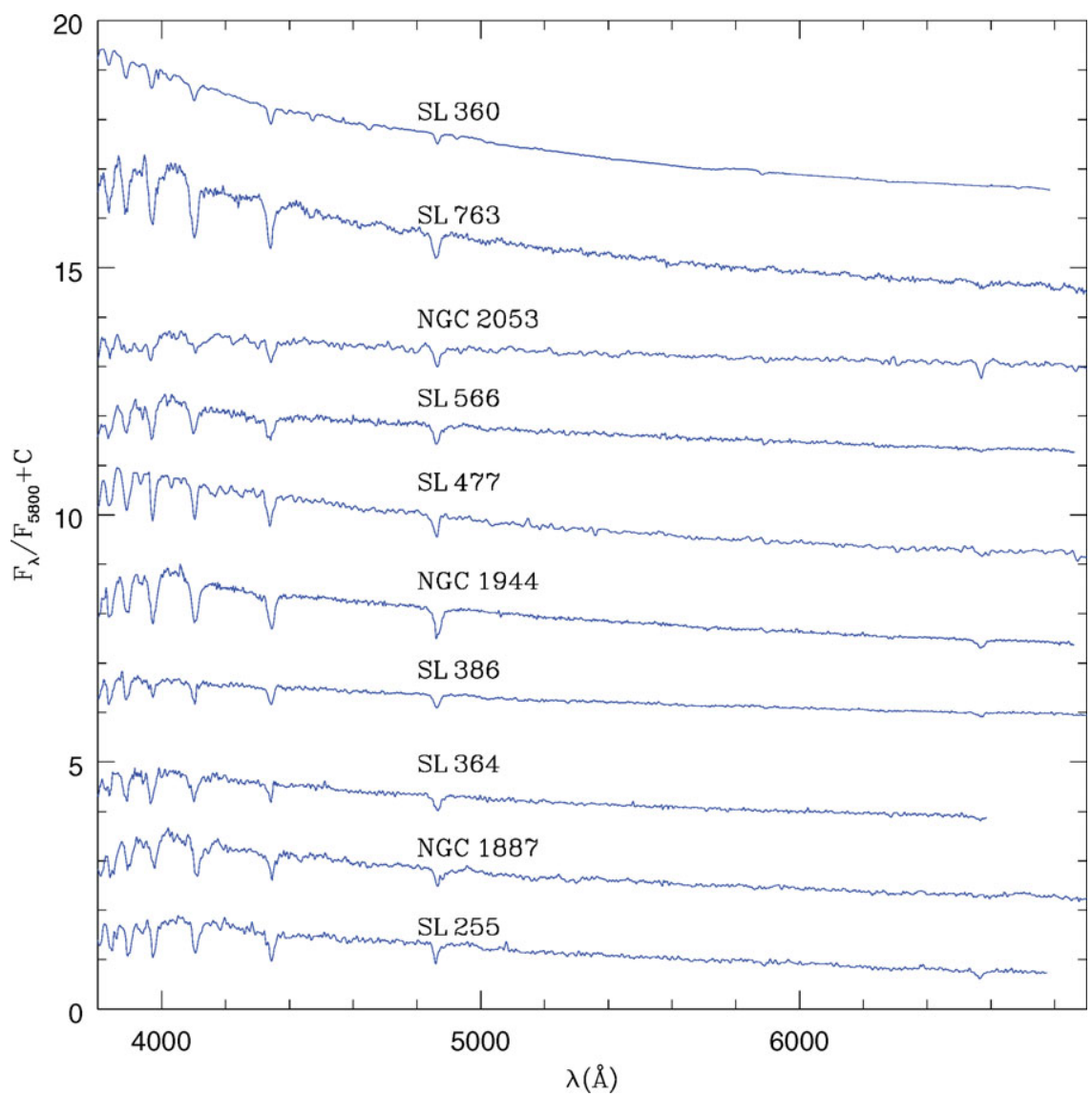

Figure 2. Observed integrated spectra of 10 LMC clusters. Spectra are in relative $F_{\lambda}$ units normalised at $\lambda \sim 5800 \AA$. Constants have been added to the spectra for clarity, except for the bottom one.

\section{Determination of cluster fundamental parameters}

Age and foreground reddening values of the clusters were simultaneously derived by means of a template matching method. This was done by achieving the best possible match between the continuum and lines of the cluster's integrated spectrum and those from an integrated spectrum with known properties (e.g., Talavera et al. 2006). A direct reddening-independent age estimate was first obtained from EWs of the Balmer absorption lines by interpolating these values in the calibration of Bica \& Alloin (1986b). The diagnostic diagrams involving the sum of EWs of selected spectral lines denoted $\mathrm{S}_{\mathrm{h}}$ and $\mathrm{S}_{\mathrm{m}}$ were employed together with the calibrations with age and metallicity by Santos \& Piatti (2004). $\mathrm{S}_{\mathrm{m}}$ corresponds to the sum of three metallic lines (K Ca II, G band and $\mathrm{Mg} \mathrm{I})$ and $\mathrm{S}_{\mathrm{h}}$ corresponds to the sum of three Balmer lines $(\mathrm{H} \beta, \mathrm{H} \gamma$ and $\mathrm{H} \delta)$. Foreground reddening $\mathrm{E}(\mathrm{B}-\mathrm{V})$ values were estimated from the template matching method and also from the interstellar extinction maps by $\mathrm{BH}$. Then, we selected an appropriate set of template spectra according to the age provided by the mentioned EWs and varied reddening and template to get the best match of continuum, Balmer and metal lines of the observed spectrum to that of the template that most resembles it. Reddening corrections were performed employing the interstellar absorption law by Seaton (1979). 
Table 3. Age and reddening determinations for SMC clusters.

\begin{tabular}{lcccccc}
\hline Cluster & $\mathbf{E}(\mathbf{B}-\mathbf{V})$ & $\begin{array}{c}\mathbf{E}(\mathbf{B}-\mathbf{V}) \\
(\mathrm{BH})\end{array}$ & $\begin{array}{c}\text { Balmer age } \\
(\mathrm{Myr})\end{array}$ & $\begin{array}{c}\mathbf{S}_{\mathrm{h}}, \mathbf{S}_{\mathrm{m}} \text { age } \\
(\mathrm{Myr})\end{array}$ & $\begin{array}{c}\text { Template age } \\
(\mathrm{Myr})\end{array}$ & $\begin{array}{c}\text { Adopted age } \\
(\mathrm{Myr})\end{array}$ \\
\hline NGC 242 & $0.08 \pm 0.03$ & 0.03 & 50 & 27 & $10-20 ; 35-65$ & $40 \pm 20$ \\
NGC 256 & $0.03 \pm 0.02$ & 0.03 & 50 & 36 & 200 & $150 \pm 50$ \\
NGC 265 & $0.03 \pm 0.02$ & 0.03 & $50-500$ & 41 & $50-110$ & $80 \pm 40$ \\
B 50 & $0.00 \pm 0.02$ & 0.03 & $<10$ & 5 & $3-5$ & $4 \pm 2$ \\
K 34 & $0.08 \pm 0.02$ & 0.03 & 50 & 95 & $100-150$ & $200 \pm 100$ \\
IC 1611 & $0.10 \pm 0.02$ & 0.06 & $50-100$ & 19 & $100-150$ & $130 \pm 30$ \\
IC 1626 & $0.11 \pm 0.02$ & 0.03 & 300 & 57 & $200-350$ & $250 \pm 50$ \\
IC 1641 & $0.04 \pm 0.01$ & 0.03 & 500 & 350 & 350 & $350 \pm 100$ \\
L 95 & $0.12 \pm 0.02$ & 0.03 & $50-100$ & 57 & 40 & $50 \pm 20$ \\
B 164 & $0.00 \pm 0.02$ & 0.03 & $\sim 10$ & 7 & $3-6 ; 12-35$ & $10 \pm 5$ \\
HW 85 & $0.01 \pm 0.01$ & 0.03 & $10-50$ & 82 & $10-20$ & $20 \pm 10$ \\
WG 1 & $0.12 \pm 0.02$ & 0.03 & $<10$ & 14 & $3-5$ & $4 \pm 2$ \\
NGC 796 & $0.06 \pm 0.02$ & 0.03 & $10-50$ & 22 & 20 & $20 \pm 10$ \\
\hline
\end{tabular}

Table 4. Age and reddening determinations for LMC clusters.

\begin{tabular}{lcccccc}
\hline Cluster & $\mathbf{E}(\mathbf{B}-\mathbf{V})$ & $\begin{array}{c}\mathbf{E}(\mathbf{B}-\mathbf{V}) \\
(\mathrm{BH})\end{array}$ & $\begin{array}{c}\text { Balmer age } \\
(\mathrm{Myr})\end{array}$ & $\begin{array}{c}\mathbf{S}_{\mathrm{h}}, \mathbf{S}_{\mathrm{m}} \text { age } \\
(\mathrm{Myr})\end{array}$ & $\begin{array}{c}\text { Template age } \\
(\mathrm{Myr})\end{array}$ & $\begin{array}{c}\text { Adopted age } \\
(\mathrm{Myr})\end{array}$ \\
\hline SL 14 & $0.18 \pm 0.02$ & 0.08 & $\leqslant 10$ & 8 & $10-20$ & $10 \pm 5$ \\
NGC 1695 & $0.16 \pm 0.02$ & 0.06 & $\sim 50$ & 70 & $50-110$ & $70 \pm 10$ \\
SL 56 & $0.05 \pm 0.02$ & 0.10 & $10-50$ & 18 & $12-40$ & $40 \pm 20$ \\
SL 58 & $0.13 \pm 0.03$ & 0.10 & $\sim 50$ & 50 & $35-65 ; 50-110$ & $65 \pm 30$ \\
SL 79 & $0.06 \pm 0.02$ & 0.03 & $\sim 100$ & 115 & 100 & $100 \pm 10$ \\
SL 76 & $0.08 \pm 0.02$ & 0.03 & $50-70$ & 28 & $12-40$ & $50 \pm 30$ \\
& $0.06 \pm 0.02$ & & & & $35-110$ & \\
NGC 1732 & $0.00 \pm 0.01$ & 0.03 & 50 & 20 & $35-110$ & $60 \pm 10$ \\
SL 116 & $0.00 \pm 0.02$ & 0.06 & $50-70$ & 34 & $35-65$ & $50 \pm 20$ \\
SL 168 & $0.01 \pm 0.01$ & 0.03 & 100 & 23 & $35-65$ & $60 \pm 20$ \\
NGC 1822 & $0.05 \pm 0.02$ & 0.04 & $50-100$ & 12 & $100-150$ & $125 \pm 25$ \\
HS 109 & $0.08 \pm 0.02$ & 0.06 & $50-100$ & 110 & $35-65$ & $70 \pm 20$ \\
SL 234 & $0.00 \pm 0.01$ & 0.06 & 50 & 24 & 50 & $60 \pm 20$ \\
SL 255 & $0.10 \pm 0.02$ & 0.10 & $10-100$ & 22 & $45-75$ & $60 \pm 10$ \\
NGC 1887 & $0.05 \pm 0.02$ & 0.04 & $30-50$ & 18 & $45-75$ & $70 \pm 20$ \\
SL 364 & $0.02 \pm 0.01$ & 0.09 & $\sim 50$ & 19 & 40 & $40 \pm 10$ \\
SL 360 & $0.10 \pm 0.02$ & 0.07 & $\sim 10$ & 05 & $3-6$ & $5 \pm 2$ \\
SL 386 & $0.17 \pm 0.02$ & 0.03 & $30-50$ & 16 & 60 & $70 \pm 20$ \\
NGC 1944 & $0.07 \pm 0.02$ & 0.07 & $50-100$ & 22 & $45-75$ & $60 \pm 10$ \\
SL 463 & $0.00 \pm 0.01$ & 0.06 & $10-50$ & 22 & $12-40$ & $50 \pm 10$ \\
& $0.01 \pm 0.02$ & & & 22 & $35-65$ & \\
SL 477 & $0.03 \pm 0.01$ & 0.07 & $10-50$ & 14 & $35-65$ & $40 \pm 20$ \\
NGC 1972 & $0.00 \pm 0.01$ & 0.07 & $30-50$ & 20 & 70 & $60 \pm 10$ \\
NGC 2000 & $0.02 \pm 0.01$ & 0.07 & $50-100$ & 42 & 40 & $50 \pm 10$ \\
NGC 1986 & $0.10 \pm 0.02$ & 0.07 & $30-50$ & 23 & $45-75$ & $50 \pm 20$ \\
SL 566 & $0.15 \pm 0.02$ & 0.09 & $10-50$ & 22 & $45-75$ & $50 \pm 10$ \\
NGC 2053 & $0.08 \pm 0.02$ & 0.06 & $\sim 50$ & 21 & $50-110$ & $70 \pm 30$ \\
SL 763 & $0.04 \pm 0.02$ & 0.08 & $50-100$ & 41 & 40 & $70 \pm 20$ \\
NGC 2140 & $0.04 \pm 0.01$ & 0.06 & $50-100$ & 60 & $50-110 ; 12-40$ & $60 \pm 20$ \\
\hline
\end{tabular}

\section{Age and reddening values}

The parameters determined for the SMC clusters are shown in Table 3. The colour excesses derived for the whole sample range from 0.00 (B 50 and B 164) to 0.12 (WG 1 and L 95), while the ages vary from $4 \mathrm{Myr}$ (B 50 and WG 1) to $350 \mathrm{Myr}$ (IC 1641). Four 
of the 13 clusters presented here were not previously studied. Table 4 shows ages and colour excesses determined for the selected 27 LMC stellar clusters. The reddening values range between 0.00 (NGC 1732, SL 116, SL 234 and NGC 1972) and 0.18 (SL 14), while the ages range from $5 \mathrm{Myr}$ (SL 360) to $125 \mathrm{Myr}$ (NGC 1822). In this sample, 17 of the 27 stellar clusters do not show previous studies, so we presented here new parameters for $63 \%$ of the sample. Within the expected uncertainties, the ages derived in the present work agree with those given in the literature.

\section{Summary}

We have estimated cluster ages and foreground interstellar reddening values from the comparison of the line strengths and continuum distribution of the cluster spectra with those of template cluster spectra with well-determined physical properties. Reddening values were also estimated by interpolation between the extinction maps of BH. A good agreement between ages and reddening values derived from both procedures was found. The ages of the LMC studied clusters range from 5 to 125 Myr while those of the SMC cluster sample range from 4 to $350 \mathrm{Myr}$. The present data constitute part of the elements to enhance the spectral libraries at the metallicity levels of the SMC and LMC star clusters.

\section{References}

Bica, E. 1988, A\&A, 195, 76

Bica, E. \& Alloin, D. 1986a, A\&A, 162, 21

Bica, E. \& Alloin, D. 1986b, A\&SAS, 66, 171

Bruck, M. T. 1976, Occasional Reports R. Obs., 1, 1

Burstein, D. \& Heiles, C. 1982, AJ, 87, $1165(\mathrm{BH})$

Hodge, P. W. \& Wright, F. W. 1974, AJ, 79, 858

Hodge, P. W. \& Sexton, J. A. 1966, AJ, 71, 363

Kontizas, M., Morgan, D. H., Hatzidimitriou, D., \& Kontizas, E. 1990, A\&AS, 84, 527

Kron, G. E. 1956, PASP, 68, 125

Lauberts, A. 1982, The ESO/Uppsala Survey of the ESO (B) Atlas, (European Southern Observatory)

Lindsay, E. M. 1958, MNRAS, 118, 172

Lyngå, G. \& Westerlund, B. E. 1963, MNRAS, 127, 31

Piatti, A. E., Bica, E., Clariá, J. J., Santos Jr., J. F. C., \& Ahumada, A. V. 2002, MNRAS, 335, 233

Pietrzyński, G., Udalski, A., Kubiak, M., Szymański, M., Woźniak, P., \& Żebruń, K. 1998, AcA, 48,175

Pietrzyński, G., Udalski, A., Kubiak, M., Szymański, M., Woźniak, P., \& Żebruń, K. 1999, AcA, 49,521

Santos Jr., J. F. C., Bica, E., Clariá, J. J., Piatti, A. E., Girardi, L. A., \& Dottori, H. 1995, MNRAS, 276, 1155

Santos Jr., J. F. C. \& Piatti, A. E. 2004, A\&A, 428, 79

Searle, L., Wilkinson, A., \& Bagnuolo, W. G. 1980, ApJ, 239, 803

Seaton, M. J. 1979, MNRAS, 187, 73p

Shapley, H. \& Lindsay, E. M. 1963, Irish Astron. J., 6, 74

Talavera, M. L., Ahumada, A. V., Clariá, J. J., Parisi, M. C., Santos Jr., J. F. C., \& Bica, E. 2006, BAAA, 49, 311

Westerlund, B. E. \& Glaspey, J. 1971, A\&A, 10, 1 